\title{
Evaluation of microvascular disturbances in rheumatic diseases by analysis of skin blood flow oscillations
}

Irina N. Makovik, Viktor V. Dremin, Angelina I. Zherebtsova, Alexander I. Krupatkin, Lyudmila S. Khakhicheva, et al.

Irina N. Makovik, Viktor V. Dremin, Angelina I. Zherebtsova, Alexander I. Krupatkin, Lyudmila S. Khakhicheva, Vadim F. Muradyan, Igor V. Meglinski, Andrey V. Dunaev, "Evaluation of microvascular disturbances in rheumatic diseases by analysis of skin blood flow oscillations," Proc. SPIE 10685, Biophotonics: Photonic Solutions for Better Health Care VI, 106854S (17 May 2018); doi: 10.1117/12.2288239 


\title{
Evaluation of microvascular disturbances in rheumatic diseases by analysis of skin blood flow oscillations
}

\author{
Irina N. Makovik ${ }^{*}$, , Viktor V. Dremin ${ }^{\mathrm{a}, \mathrm{d}}$, Angelina I. Zherebtsova ${ }^{\mathrm{a}}$, Alexander I. Krupatkin ${ }^{\mathrm{b}}$, \\ Lyudmila S. Khakhicheva ${ }^{c}$, Vadim F. Muradyan ${ }^{\mathrm{c}}$, Igor V. Meglinski ${ }^{\mathrm{d}, \mathrm{a}}$, Andrey V. Dunaev ${ }^{\mathrm{a}}$ \\ ${ }^{a}$ Research and Development Center of Biomedical Photonics, Orel State University, Orel, 302026, \\ Russia; \\ ${ }^{\mathrm{b}}$ Priorov National Medical Research Center of Traumatology and Orthopaedics, Moscow, 127299, \\ Russia; \\ ${ }^{\mathrm{c}}$ Orel Regional Clinical Hospital, Orel, 302028, Russia \\ ${ }^{\mathrm{d}}$ Opto-Electronics and Measurement Techniques, University of Oulu, Oulu, 90014, Finland
}

\begin{abstract}
Laser Doppler flowmetry (LDF), tissue reflectance oximetry (TRO) and pulse oximetry (PO) and cold pressor test (CPT) were used to assess the microcirculation parameters and the activation of regulatory mechanisms. LDF and TRO samples wavelet transform in the frequency bands $0.01-2 \mathrm{~Hz}$ was used to evaluate microvascular disturbances in rheumatic diseases and to assess the vascular involvement in the pathological process. The spectral components of LDF and TRO signals associated with endothelial, adrenergic, intrinsic smooth muscle, respiratory and cardiac activities were analyzed. Significant difference between healthy and rheumatology subjects was identified in perfusion parameters. Spectral analysis of the LDF signal revealed significant difference between two group of high $(>0.1 \mathrm{~Hz})$ frequency pulsations. Based on the analysed of the perfusion and amplitudes oscillation in the frequency band the decision rule for detection microvascular disturbances were synthesized. The perfusion parameter and amplitude oscillation associated with cardiac activities included in the decision rule. Based on the measured parameters and the result of wavelet transform LDF- and TRO-signals the parameters for detection of complications associated with microvascular disturbances and their possible causes were proposed.
\end{abstract}

Keywords: laser Doppler flowmetry, tissue reflectance oximetry, pulse oximetry, wavelet transform, microvascular disturbances, rheumatic diseases.

\section{INTRODUCTION}

According to international statistics, $8.4 \%$ of women and $5 \%$ of men suffer from rheumatic diseases (RD) ${ }^{1}$. RD represents a large group of nosological forms of various origin. Unifying factors of all rheumatic diseases are the localization of the main pathological process in connective tissue and their clinical manifestation in the form of joint syndrome. Development of RD causes damage to the internal organs, which, together with the main manifestations of the disease, leads to disability in the first ten years of the disease course, loss of work ability and decrease life expectancy ${ }^{1}$.

Microvascular disturbances are unifying link in the pathogenesis of all RD. These disturbances are manifested in deviations and abnormalities in vascular tone regulation, disorders of blood microcirculation and morphological microvascular structure changes. Among the most common clinical forms of microvascular disturbances manifestation are increasing vascular wall permeability, formation of vasculitis, mucoid swelling of the vessels wall with a narrowing of their lumen, symmetrical paroxysmal spasm of arteries, arterioles caused by cold, emotional stress and dilatations of the capillary network, changes in the density of capillaries, their asymmetry, and the formation of branching capillaries, megacapillaries, microhemorrhagia and avascular regions ${ }^{2-4}$.

Development of these microvascular disturbances cause an imbalance in the concentrations of the main chromophores of the skin (oxyhemoglobin and deoxyhemoglobin) and destructuring of components of the vascular wall. The combination of these processes provokes the development of complications, which cause the development of hypoxia, edema of

\footnotetext{
*Correspondence: e-mail: irina.makovik@gmail.com; phone: 74862 419837; www.bmecenter.ru/en
}

Biophotonics: Photonic Solutions for Better Health Care VI, edited by Jürgen Popp,

Valery V. Tuchin, Francesco Saverio Pavone, Proc. of SPIE Vol. 10685, 106854S

(C) 2018 SPIE · CCC code: $0277-786$ X/18/\$18 $\cdot$ doi: $10.1117 / 12.2288239$ 
tissues and the appearance of necrobiotic processes ${ }^{5}$. The involvement of vessels in the pathological process can lead to aggravation of the disease and in most cases influences the strategy of treatment and prognosis of the disease in general.

To study microvascular system functional state in vivo different non-invasive methods of optical reflectance spectroscopy $^{6}$, optical coherence tomography $(\mathrm{OCT})^{7}$, laser speckle imaging ${ }^{8}$, laser Doppler flowmetry (LDF) ${ }^{9}$, polarization-based imaging ${ }^{10}$ and other have been applied. The LDF is based on the probing of biological tissue by using laser radiation and the extraction of a phase shift (Doppler shift) of registration signal. This signal is formed as a result of reflect and scatter light from moving red blood cells and is determined by the speed of their movement ${ }^{11}$. The main parameter recorded by the LDF is perfusion $\left(I_{m}\right)$.

For evaluation of the skin main chromophores (oxyhemoglobin and deoxyhemoglobin) tissue reflectance oximetry (TRO) and pulse oximetry (PO) are applied. The TRO is based on measuring different hemoglobin fractions spectrophotometrically. The main parameter recorded by the TRO is tissue oxygen saturation $\left(S_{t} O_{2}\right)$. This parameter describes the percentage of oxyhaemoglobin on the background of the all haemoglobin fractions (oxyhaemoglobin and deoxyhaemoglobin) sum. The PO registers percent oxyhemoglobin (oxygen saturation) in the arterial blood $\left(\mathrm{S}_{a} \mathrm{O}_{2}\right)$.

The LDF and TRO together with the wavelet transform are capable of providing vast amounts of physiological data. This data comes in the form of frequency bands $(0.0095-1.6 \mathrm{~Hz})$, which reflect the different regulation mechanisms of skin blood flow ${ }^{12}$. Oscillations of skin blood flow observed in the frequency band 0.6 to $1.6 \mathrm{~Hz}$ and 0.2 to $0.6 \mathrm{~Hz}$ are associated with heart and respiratory activities ${ }^{13}$, respectively, whereas oscillations in the 0.0095 to $0.1 \mathrm{~Hz}$ frequency band are related to myogenic ${ }^{14}$, neurogenic ${ }^{14}$, and endothelial activities ${ }^{16}$.

Additional possibilities in the study of microcirculatory bed and in the evaluation of microvascular disturbances are the use of functional tests, for example, cold pressor test, occlusion test, respiratory, and etc. The cold pressor test (CPT) application is perspective for the evaluate of microcirculatory disturbances. The choice of this functional test is due to the fact that during $\mathrm{RD}$ a failure in response to cold stimulation of a vasomotor center is observed, which leads either to an increased spasm of the vessels, or to vasodilation.

For the more intensive introduction of diagnostic methods into clinical practice not only the development of study protocols but and the detection of diagnostically parameters, which could be used for the development of decisive rules for revealing of presence or absence of various disorders are important.

The aim of this work is a detection of diagnostic parameters obtained within the studies utilizing LDF, TRO, and PO techniques, CPT and wavelet transform of LDF and TRO signals, as well as the development of decisive rule for detection of microvascular disturbances and associated complications.

\section{MATERIAL AND METHODS}

\subsection{Study protocol}

Study protocol consisted in the registration of microcirculatory bed parameters using LDF, TRO and PO before and after cooling (Table 1). Cooling during CPT was carried out for 5 min, patient's arms were submerged in cold water.

\begin{tabular}{|c|c|c|c|c|c|}
\hline Basic test & BT1 & $\begin{array}{c}\text { Cooling } \\
\mathrm{T}_{\text {water }}=15^{\circ} \mathrm{C}\end{array}$ & BT2 & $\begin{array}{c}\text { Relaxation } \\
\text { period }\end{array}$ & BT3 \\
\hline Stage duration, $\min$ & 5 & 5 & 5 & 15 & 5 \\
\hline Study duration, min & \multicolumn{5}{|c|}{35} \\
\hline
\end{tabular}

\subsection{Measured and calculated parameters}

During of the studies LDF, TRO and PO signals were measurement. The obtained LDF and TRO signals were decomposed using a wavelet transform ${ }^{17}$. The wavelet coefficients were counted for the frequency band 0.01 to $2 \mathrm{~Hz}$. Power spectral density was calculated for three records of LDFs and TROs for each patient. The integral wavelet spectra were averaged over the group of patients. The energy distribution for every frequency band has been calculated.

Myogenic tone $(M T)$ and rate of oxygen consumption $(O C)$ were used for detection of complications associated with microvascular disturbances. These parameters were calculated by the method presented in the article ${ }^{18}$ with using the 
measured parameters and the results of LDF and TRO signals wavelet transform. Analysis of $M T$ and $O C$ allows to evaluate the tone oscillatory component of pre-capillaries and precapillary sphincters, that determines perfusion in the nutritive bed, as well as quantitatively describes the oxygen diffusion through micro-vessels walls.

The analysis changes of $M T$ and $A_{c} / A_{r}$ ratio during the study process was proposed to use for detection of possible causes of complications associated with microvascular disturbances. $A_{c} / A_{r}$ is calculated by the results of the LDF signals wavelet transform and allows to evaluate the ratio of the arterial blood flow to venular.

The data processing was performed by Mathematica 8.0, Wolfram research. Statistical analysis of the measured and calculated parameters was performed using non-parametric criteria. The Mann-Whitney test was used for comparing values between groups. The Wilcoxon test was used for comparing values within a single group.

\subsection{Experimental equipment and study conditions}

Experimental systems "LAKK-OP" and "LAKK-M" (SPE "LAZMA" Ltd., Russia) were applied for the measurement LDF and TRO signals. Temperature of water was controlled by a contactless digital thermometer (Sensitec NB401, Netherlands).

All measurements were performed in conditions of physical and mental rest $2 \mathrm{~h}$ after a meal. Volunteers also underwent a preliminary adaptation to room temperature $24-25^{\circ} \mathrm{C}$ for $15-20 \mathrm{~min}$ in a sitting position, with the right arm on the table at heart level. The adaptation of volunteers to standard room temperature and abidance of study protocol during all measurements reduce the influence of different factors on results of diagnosis.

Schemes of experimental installation during the measurement, during the cold exposure and schematic presentation of the LDF and TRO probe and PO sensor positioning on a finger are showed in Fig.1.

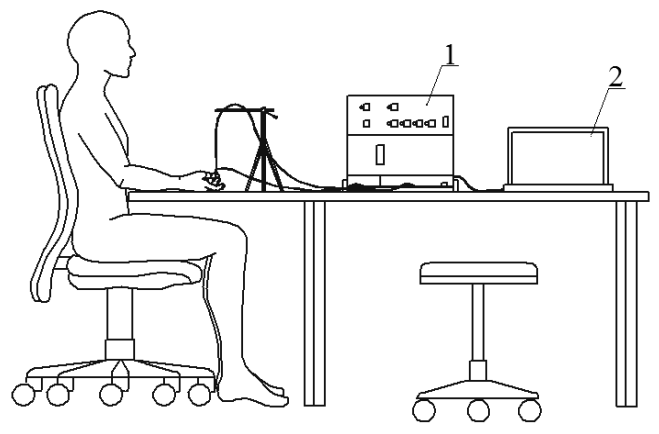

a)

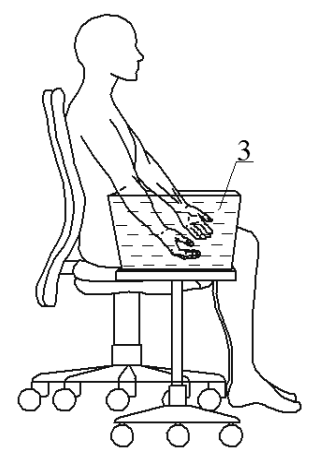

b)

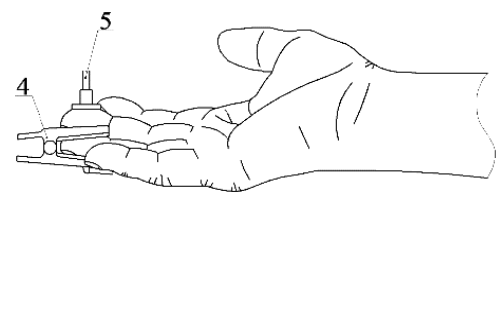

c)

Figure 1. Schemes of experimental installation during the measurement (a), during the cold exposure (b) and schematic presentation of the LDF probe positioning on a finger $(c)$ : 1 - experimental equipment, 2 - computer, 3 - container with water, 4 - PO sensor, 5 - LDF and TRO probe.

\subsection{Study groups}

The study was carry out on 32 (16 male and 16 female) healthy volunteers (HV) (average age $22 \pm 2$ year) and 60 (12 male and 48 female) patient with rheumatic diseases (PRD) (average age $55 \pm 14$ year) from the Rheumatology Department of the Orel Regional Clinical Hospital, Russia. The study was approved by the local Committee for Human Biomedical Research Ethics, and all subjects signed an informed consent.

The PRD includes individuals with the following diseases: rheumatoid arthritis $(\mathrm{n}=31)$, systemic lupus erythematosus $(\mathrm{n}=8)$, primary gonarthrosis, bilateral $(\mathrm{n}=6)$, ankylosing spondylitis $(\mathrm{n}=3)$, gout $(\mathrm{n}=3)$, psoriatic arthritis $(\mathrm{n}=2)$, systemic scleroderma $(\mathrm{n}=2)$, primary osteoarthritis $(\mathrm{n}=1)$, chronic gouty arthritis $(\mathrm{n}=1)$, chronic rheumatic heart disease $(\mathrm{n}=1)$, idiopathic dermatopolymiositis $(\mathrm{n}=1)$, primary generalized osteoarthritis $(\mathrm{n}=1)$, spondylitis seronegative $(\mathrm{n}=1)$.

The main characteristics of PRD are presented in Table 2. The data-range averages, as well as the minimum and maximum values, are provided for each parameter. 
Table 2. Main characteristics of PRD

\begin{tabular}{|c|c|}
\hline Name of the parameter & Value \\
\hline Disease longitude, years & $9(0.1-36)$ \\
Systolic pressure, mmHg & $129(105-160)$ \\
Diastolic pressure, mmHg & $74(56-84)$ \\
Pulse/min & $81(70-90)$ \\
RF, IU/ml & $121(8-512)$ \\
$\mathrm{CRP}, \mathrm{mg} / 1$ & $52(6-192)$ \\
$\mathrm{RBC}, 10^{12} / 1$ & $4.4(3.5-6.7)$ \\
$\mathrm{HGB}, \mathrm{g} / 1$ & $128(91-155)$ \\
$\mathrm{MCHC}$ & $0.9(0.7-1.0)$ \\
$\mathrm{PLT}, 10^{9} / 1$ & $334(118-385)$ \\
$\mathrm{WBC}, 109 / 1$ & $7.5(3.0-18.7)$ \\
$\mathrm{LYM}, \%$ & $31(6-83)$ \\
$\mathrm{MONO}, \%$ & $4.4(1.0-31.0)$ \\
$\mathrm{ESR}, \mathrm{mm} / \mathrm{h}$ & $23(2.0-62)$ \\
\hline
\end{tabular}

Notes: RF - rheumatoid factor; CRP - C-reactive protein; RBC - red blood cells; HGB - haemoglobin; MCHC - mean corpuscular haemoglobin concentration; PLT - platelets; WBC - white blood cells; LYM - lymphocyte; MONO - monocyte; ESR - erythrocyte sedimentation rate

\section{EXPERIMENTAL RESULTS AND DISCUSSION}

Experimental studies results showed that CPT evokes different reaction from the microcirculatory bed in HV and PRD. Figure 2 presents the results of perfusion change during CPT and averaged over both groups wavelet spectra in BT1 ${ }^{19}$.

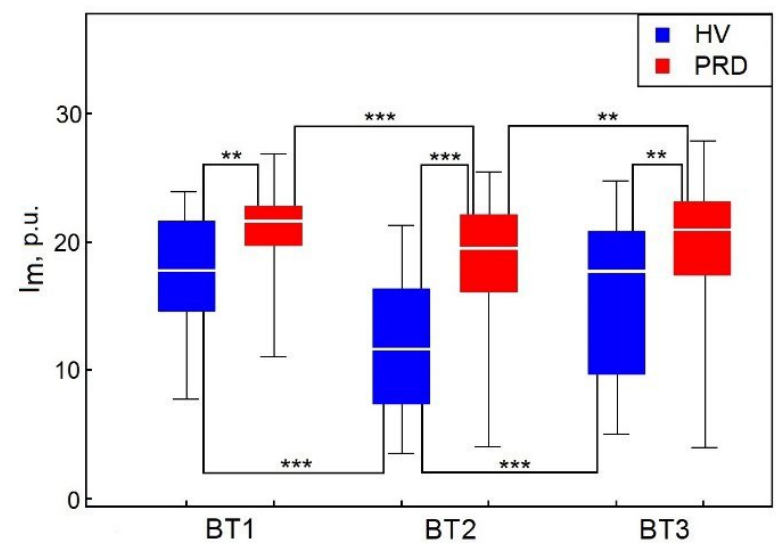

a)

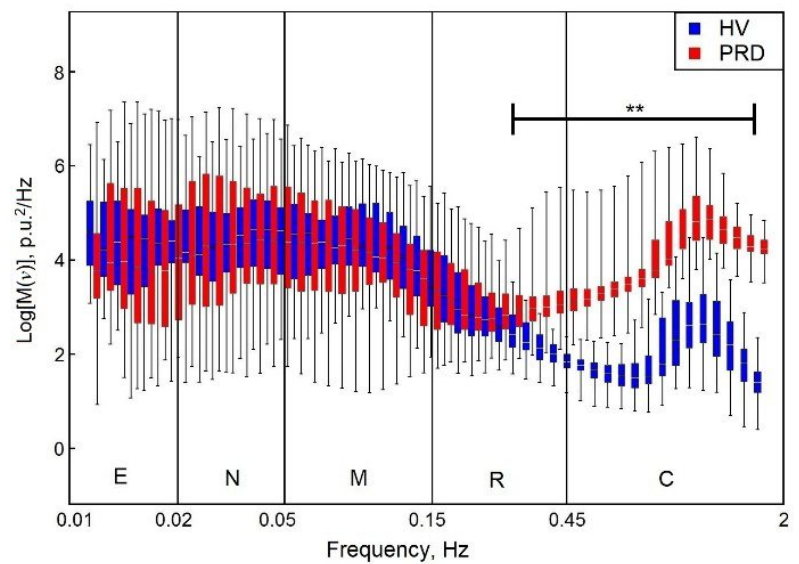

b)

Figure 2. (a) Analysis results of the perfusion in BT1, BT2 and BT3; (b) power spectral density in BT1. Note: Statistically significant differences in the values with ${ }^{* *} \mathrm{p}<0.01 ; * * \mathrm{p}<0.005 ; \mathrm{E}-$ endothelial, $\mathrm{N}$ - neurogenic, $\mathrm{M}-$ myogenic, $\mathrm{R}-$ respiratory, $\mathrm{C}-$ cardiac frequency bands of blood flow oscillations.

From Fig.2(a) one can see that throughout the study, significant differences in perfusion between the study groups were observed, herewith PRD has higher perfusion in basal state then HV. The cooling provokes the vasoconstriction which leads to the perfusion decrease. The vasoconstriction is more evident in HV.

Analysis of averaged over HV and PRD wavelet spectra in BT1 showed high frequency pulsations of the blood flow have larger energy in PRD than in HV, herewith the difference is significant above the frequency of $0.24 \mathrm{~Hz}(\mathrm{p}<0.01)$. Immediately after the cooling the difference between HV and PRD in the low-frequency part of the spectra doesn't observe, at the same time the difference in the energy of high frequency pulsations becomes more significant. After 20 minutes a total restore of blood flow, as far as spectral composition of the LDF signals, have been observed. 
As was previously stated in the work ${ }^{19}$ this reaction is associated with a weak damping capacity of the vascular bed due to a decrease in the elasticity of the vascular wall and increase its stiffness, and also because of morphological disturbances arising during RD (formation of megacapillaries, thinning of the capillary network).

Analysis of MT and OC between the study groups revealed the significance of their differences in BT3.

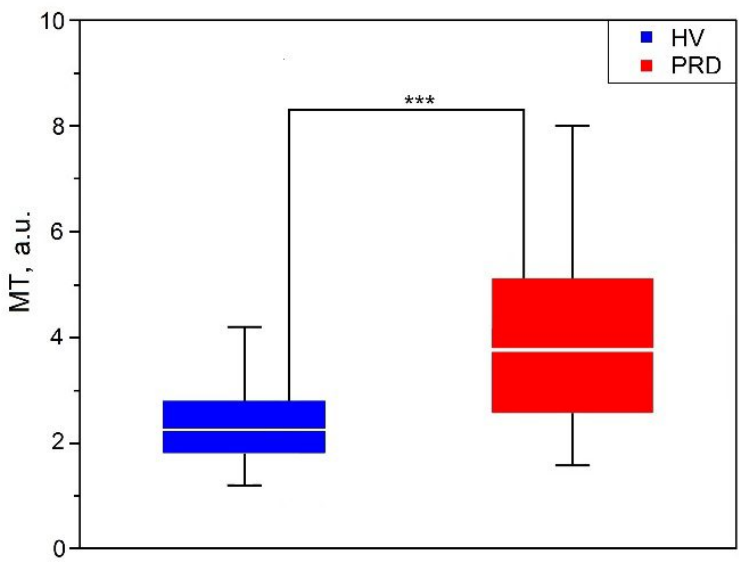

a)

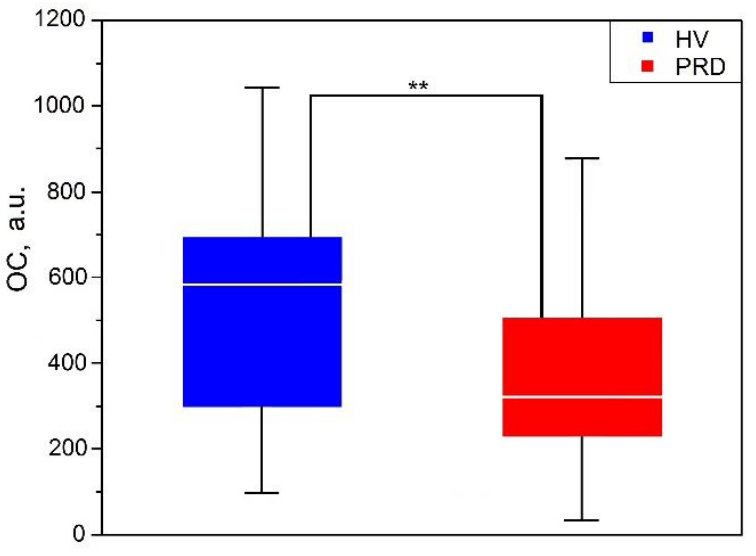

b)

Figure 3. Analysis results of the myogenic tone (a) and rate of oxygen consumption (b) in BT3. Notes: Statistically significant differences in the values with $* * \mathrm{p}<0.01 ; * * \mathrm{p}<0.005$.

A higher level of $M T$ in PRD (Fig.3a) indicates the closure of precapillary sphincters and the decrease of blood circulation in capillaries. The increase of $M T$ and decrease of $I_{m}$ regarding their level in BT1 is a sign of increased of the vascular wall spasmodic.

Differences in $O C$ and decrease of this parameter in PRD (Fig.3b) indicate the possible violations of the microvascular bed surface of the smallest arterioles and capillaries. The decrease of $O C$ against the background of $M T$ increase is a sign of reduction of oxygen diffused through the vessels walls. These processes could lead to hypoxia, edema of tissues and the appearance of necrobiotic processes at untimely diagnostics and absence of treatment.

An analysis of the possible causes of these pathological changes revealed their association with an increase of myogenic tone, as well as its combination with venous stasis. So $M T, O C$, and also $A_{c} / A_{r}$ can be used as an additional diagnostic criterias for detection of complications associated with microvascular disturbances and their possible causes.

Based on the obtained results and on the differences between groups, the perfusion values and the maximum amplitude oscillations during CPT were used to the synthesis of the decision rule for diagnose microcirculatory disorders in RD. These parameters satisfy the principles of statistical independence, as well as the significance of the differences of their values, calculated for the PRD and HV.

Linear discriminant analysis was choose for synthesis of the decision rule. This method is easy to implement and allows to get the final result in the form of a discriminant function with high quality of the classification. Linear discriminant analysis proved itself well for classification of medical data ${ }^{9,20}$. The discriminant function is synthesized in such a way as to provide high sensitivity while providing excellent specificity. The sensitivity and specificity for the classification rule for a different combination of measured parameters are presented in the Table 3.

As can be seen from the Table 3, the lowest level of error is obtained with the combination of $I_{m}$ and amplitude of pulse wave immediately after cooling (BT2). Fig.4(a) shows the scatter plot of the experimental data with applied discriminant line that divides the experimental points into two groups (HV, PRD). 
Table 3. Sensitivity and specificity for the classification rule.

\begin{tabular}{|l|c|c|c|c|c|c|c|c|c|c|c|c|}
\hline Parameter & $I_{m 1}$, & $I_{m 1}$, & $I_{m 1}$, & $I_{m 1}$, & $I_{m 1}$, & $I_{m 2}$, & $I_{m 2}$, & $I_{m 2}$, & $I_{m 3}$, & $I_{m 3}$, & $I_{m 3}$, & $I_{m 3}$, \\
\hline Sensitivity & $A_{e 1}$ & $A_{n 1}$ & $A_{m 1}$ & $A_{r 1}$ & $A_{c 1}$ & $A_{m 2}$ & $A_{r 2}$ & $A_{c 2}$ & $A_{n 3}$ & $A_{m 3}$ & $A_{r 3}$ & $A_{c 3}$ \\
\hline Specificity & 0.72 & 0.75 & 0.72 & 0.80 & 0.85 & 0.78 & 0.83 & 0.92 & 0.73 & 0.72 & 0.75 & 0.90 \\
\hline
\end{tabular}

The immediate diagnostic criterion, with the aid of the discriminant function, allows relating of a newly measured subject to one of the two groups:

$$
f(x)=0.12 I_{m 2}+2.93 A_{c 2}-3.25 \text {. }
$$

From Fig.4(a) one can see that the shift to the right characterizes the transition to the state of the presence of microcirculatory disorders.

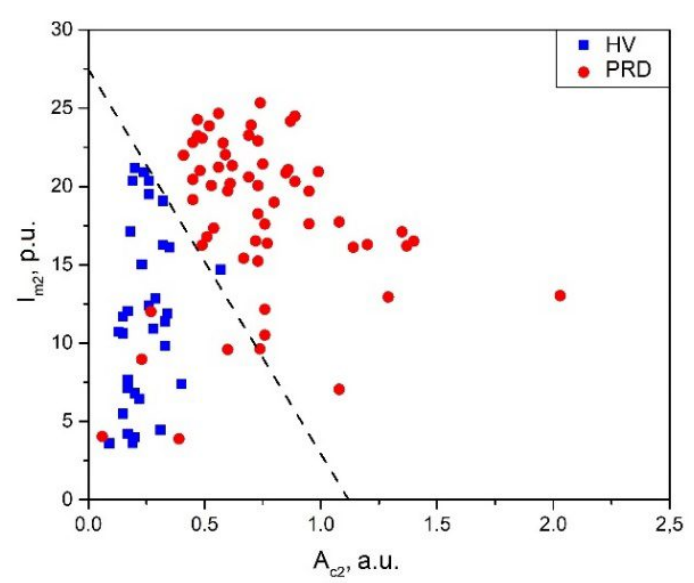

a)

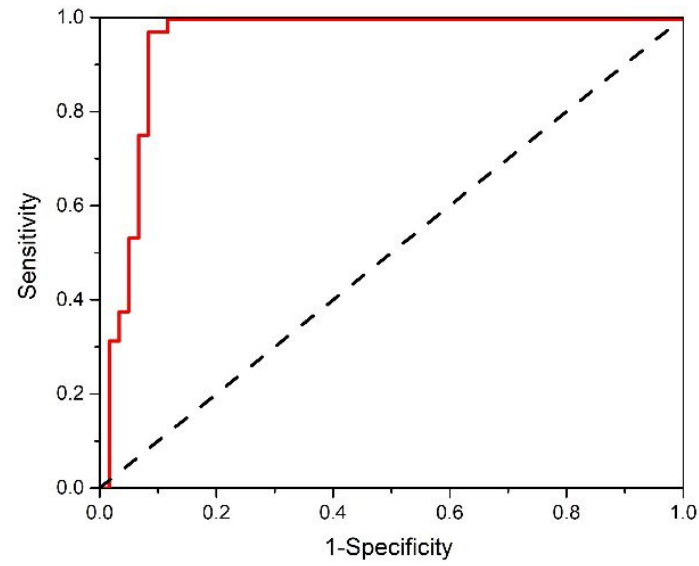

b)

Figure 4. (a) The scatter plot with applied discriminant lines, obtained by linear discriminant analysis method and (b) ROCcurves for assessing the effectiveness of the classifiers.

Figure 4(b) shows the ROC curve calculated for the obtained discriminant function. Area under curve (AUC) was used for compare the quality of different classifying rules. For the synthesized decision rule AUC $=0.92$. This indicator indicates a high level of quality of the classifier. Thus, the perfusion and amplitude of the pulse wave can act as markers for microcirculatory disorders in RD.

\section{CONCLUSION}

The proposed approach that based on application of LDF, TRO and PO during a CPT and with the following wavelet transform of LDF and TRO signals could be used for evaluation of microvascular disturbances in RD and associated complications. Spectral properties of cutaneous blood flow vary due to the pathological process in the microcirculation system. The developed technique, proposed criteria and the decision rule have high sensitivity and specificity and can be used in clinical application for functional evaluation of blood flow regulation and diagnosis of microvascular disturbances associated with RD.

\section{ACKNOWLEDGMENTS}

The work was supported by the Russian Foundation for basic research (project no. 17-41-590560) and by grant of the President of the Russian Federation for state support of young Russian scientists № MK-3400.2018.8. 


\section{REFERENCES}

[1] Crowson, C. S., Matteson, E. L., Myasoedova, E., Michet, C. J., Ernste, F. C., Warrington, K. J., Davis, J. M., Hunder, G. G., Therneau, T. M. and Gabriel, S. E., "The lifetime risk of adult-onset rheumatoid arthritis and other inflammatory autoimmune rheumatic diseases," Arthritis \& Rheumatology 63(3), 633-639 (2011).

[2] Szekanecz, Z. and Koch, A. E., "Vascular involvement in rheumatic diseases: 'vascular rheumatology'," Arthritis Research \& Therapy 10(5), 224 (2008).

[3] Heidrich, H., "Functional vascular diseases: Raynaud's syndrome, acrocyanosis and erythromelalgia," VASA. Supplementum 39(1), 33 (2010).

[4] Gutierrez, M., De Angelis, R., Bertolazzi, C. and Grassi, W., "Capillaroscopic scleroderma-like pattern in patients without connective tissue disorders," Rheumatology 49(10), 1994-1996 (2010).

[5] Flavahan, N. A., "A vascular mechanistic approach to understanding Raynaud phenomenon," Nat. Rev. Rheumatol. 11(3), 146-158 (2015).

[6] Thorn, C.E., Matcher, S.J., Meglinski, I., and Shore, A.C., "Is mean blood saturation a useful marker of tissue oxygenation?,” Am. J. Physiol. Heart Circ. Physiol. 296(5), H1289-H1295 (2009).

[7] Doronin, A. and Meglinski, I., "Imaging of subcutaneous microcirculation vascular network by double correlation Optical Coherence Tomography," Laser \& Photonics Reviews 7(5), 797-800 (2013).

[8] Kalchenko, V., Kuznetsov, Y., Meglinski, I. and Harmelin, A., "Label free in vivo laser speckle imaging of blood and lymph vessels,” J. Biomed. Opt. 17(5), 050502 (2012).

[9] Zherebtsov, E.A., Zherebtsova, A.I., Doronin, A., Dunaev, A.V., Podmasteryev, K.V., Bykov, A., Meglinski, I. "Combined use of laser Doppler flowmetry and skin thermometry for functional diagnostics of intradermal finger vessels," J. Biomed. Opt. 22(4), 040502 (2017).

[10] Doronin, A., Tchvialeva, L., Markhvida, I., Lee, T., and Meglinski, I., "Backscattering of linearly polarized light from turbid tissue-like scattering medium with rough surface," J. Biomed. Opt. 21(7), 071117 (2016).

[11] Fredriksson, I., Larsson, M. and Strömberg, T., "Model-based quantitative laser Doppler flowmetry in skin," J. Biomed. Opt. 15(5), 057002 (2010).

[12] Rossi, M., Carpi, A., Galetta, F., Franzoni, F. and Santoro, G., "Skin vasomotion investigation: a useful tool for clinical evaluation of microvascular endothelial function?," Biomed Pharmacother 62(8), 541-554 (2008).

[13] Krupatkin, A. I., "Cardiac and respiratory oscillations of the blood flow in microvessels of the human skin," Human Physiology 34(3), 323-329 (2008).

[14] Krupatkin, A. I., "Blood flow oscillations at a frequency of about $0.1 \mathrm{~Hz}$ in skin microvessels do not reflect the sympathetic regulation of their tone," Human Physiology 35(2), 183-191 (2009).

[15] Soderstrom, T., Stefanovska, A., Veber, M. and Svensson, H., "Involvement of sympathetic nerve activity in skin blood flow oscillations in humans," Am. J. Physiol. Heart. Circ. Physiol. 284(5), H1638-46 (2003).

[16] Kvandal, P., Landsverk, S. A., Bernjak, A., Stefanovska, A., Kvernmo, H. D. and Kirkeboen, K. A., "Lowfrequency oscillations of the laser Doppler perfusion signal in human skin," Microvasc. Res. 72(3), 120-7 (2006).

[17] Frick, P., Mizeva, I. and Podtaev, S., "Skin temperature variations as a tracer of microvessel tone," Biomed. Signal. Process. Control. 21, 1-7 (2015).

[18] Makovik, I. N., Dunaev, A. V., Dremin, V. V., Krupatkin, A. I., Sidorov, V. V., Khakhicheva, L. S., Muradyan, V. F., Pilipenko, O. V., Rafailov, I. E. and Litvinova, K. S., "Detection of angiospastic disorders in the microcirculatory bed using laser diagnostics technologies," J. Innov. Opt. Health. Sci. 11(1), 1750016 (2017).

[19] Mizeva, I., Makovik, I., Dunaev, A., Krupatkin, A. and Meglinski, I., "Analysis of skin blood microflow oscillations in patients with rheumatic diseases," J. Biomed. Opt. 22(7), 70501 (2017).

[20] Dremin, V., Zherebtsov, E., V., S., Krupatkin, A., Makovik, I., Zherebtsova, A., Zharkikh, E., Potapova, E., Dunaev, A., Doronin, A., Bykov, A., Rafailov, I., Litvinova, K., Sokolovski, S. and E., R., "Multimodal optical measurement for study of lower limb tissue viability in patients with diabetesmellitus," J. Biomed. Opt. 22(8), 085003 (2017). 